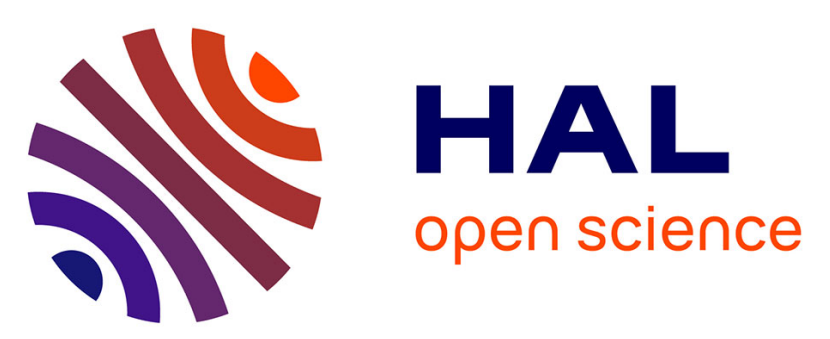

\title{
Minimum Convex Piecewise Linear Cost Tension Problem on Quasi-k Series-Parallel Graphs
}

Bruno Bachelet, Philippe Mahey

\section{To cite this version:}

Bruno Bachelet, Philippe Mahey. Minimum Convex Piecewise Linear Cost Tension Problem on Quasik Series-Parallel Graphs. 4OR: A Quarterly Journal of Operations Research, 2004, 2 (4), pp.275-291. 10.1007/s10288-004-0049-3 . hal-00107057

\section{HAL Id: hal-00107057 https://hal.science/hal-00107057}

Submitted on 16 Jan 2019

HAL is a multi-disciplinary open access archive for the deposit and dissemination of scientific research documents, whether they are published or not. The documents may come from teaching and research institutions in France or abroad, or from public or private research centers.
L'archive ouverte pluridisciplinaire HAL, est destinée au dépôt et à la diffusion de documents scientifiques de niveau recherche, publiés ou non, émanant des établissements d'enseignement et de recherche français ou étrangers, des laboratoires publics ou privés. 


\title{
Minimum Convex Piecewise Linear Cost Tension Problem on Quasi Series-Parallel Graphs
}

\author{
Bruno Bachelet ${ }^{1}$ and Philippe Mahey ${ }^{2}$ \\ LIMOS, UMR 6158-CNRS, \\ Université Blaise-Pascal, BP 10125, 63173 Aubière, France.
}

Research Report LIMOS/RR03-19

${ }^{1}$ bachelet@isima.fr - http://frog.isima.fr/bruno

${ }^{2}$ mahey@isima.fr 


\begin{abstract}
This article proposes an extension, combined with the out-of-kilter technique, of the aggregation method (that solves the minimum convex piecewise linear cost tension problem, or CPLCT, on series-parallel graphs) to solve CPLCT on quasi series-parallel graphs. To make this algorithm efficient, the key point is to find a "good" way of decomposing the graph into series-parallel subgraphs. Decomposition techniques, based on the recognition of series-parallel graphs, are thoroughly discussed.
\end{abstract}

Keywords: minimum cost tension, series-parallel graph, graph decomposition, series-parallel recognition.

\title{
Résumé
}

Cet article propose une extension, combinée avec la technique de mise-à-conformité, de la méthode d'agrégation (qui résout le problème de tension minimum à coûts convexes linéaires par morceaux, ou CPLCT, sur des graphes série-parallèles) afin de résoudre CPLCT sur des graphes quasi série-parallèles. Pour rendre cet algorithme efficace, le point clé est de trouver une "bonne" décomposition du graphe en sous-graphes série-parallèles. Des techniques de décomposition, basées sur la reconnaissance de graphes série-parallèles, sont discutées en détails.

Mots clés : tension de coût minimum, graphe série-parallèle, décomposition de graphe, reconnaissance de graphe série-parallèle.

\section{Acknowledgements / Remerciements}

This project was partially funded by France-Brazil cooperation project CAPES-COFECUB 398/02. 


\begin{abstract}
This article proposes an extension, combined with the out-of-kilter technique, of the aggregation method (that solves the minimum convex piecewise linear cost tension problem, or CPLCT, on series-parallel graphs) to solve CPLCT on quasi series-parallel graphs. To make this algorithm efficient, the key point is to find a "good" way of decomposing the graph into series-parallel subgraphs. Decomposition techniques, based on the recognition of seriesparallel graphs, are thoroughly discussed.
\end{abstract}

Keywords: minimum cost tension, series-parallel graph, graph decomposition, seriesparallel recognition.

\title{
Introduction
}

[4] proposed an aggregation method to solve the minimum convex piecewise linear cost tension problem (or CPLCT) on series-parallel graphs (or SP-graphs). It was shown to be competitive on this class of graphs with the best dual cost-scaling algorithms (see [1]). This article proposes to combine the aggregation method with the out-of-kilter approach (cf. [10]) to provide an efficient method to solve CPLCT on a slightly more general family of graphs called quasi series-parallel.

Let $\pi: X \longmapsto \mathbb{R}$ be a function that assigns a potential to each node of the graph $G=(X ; U)$. Let $m=|U|$ and $n=|X|$. The tension $\theta_{u}$ of an arc $u=(x ; y)$ is the difference of potentials $\theta_{u}=\pi_{y}-\pi_{x}$ and is constrained to $\theta_{u} \in\left[a_{u} ; b_{u}\right] \subset \mathbb{R}$. CPLCT can be modeled as a linear program:

$$
(P)\left\{\begin{array}{l}
\operatorname{minimize} \sum_{u \in U} c_{u}\left(\theta_{u}\right) \\
\text { with } \quad \pi_{y}-\pi_{x}=\theta_{(x ; y)}, \forall(x ; y) \in U \\
a_{u} \leq \theta_{u} \leq b_{u}, \forall u \in U
\end{array}\right.
$$

where $c_{u}$ are convex piecewise linear functions. In this article, they will be defined as follows:

$$
c_{u}\left(\theta_{u}\right)=\left\{\begin{array}{l}
c_{u}^{1}\left(o_{u}-\theta_{u}\right), \text { if } \theta_{u}<o_{u} \\
c_{u}^{2}\left(\theta_{u}-o_{u}\right), \text { if } \theta_{u} \geq o_{u}
\end{array}\right.
$$

This problem is formally related to a minimum cost flow problem by duality (cf. [1]). It arises for instance in the synchronization of hypermedia documents where each document $u$ has an elastic duration $\theta_{u}$ that can be adjusted around a referential value $o_{u}$ (see [6]).

Section 1 proposes an overview of the SP-graphs and the aggregation method. It also defines quasi SP-graphs, that are not perfectly series-parallel. Section 2 presents an extension of the aggregation technique, called reconstruction, for quasi SP-graphs. To be efficient, this new algorithm rely on a "good" decomposition of the graph into SP-subgraphs. This is the key point of the procedure discussed in Sections 3 and 4. Section 5 presents comparative numerical results.

\section{Aggregation Method}

\subsection{Series-Parallel Graphs}

A common definition of series-parallel graphs is based on a recursive construction of the graphs (e.g. [8], [9], [13]) that is very intuitive and close to the way synchronization constraints are built in a hypermedia document. A graph is series-parallel, also called SP-graph, if it is obtained from a graph with only two nodes linked by an arc, applying recursively the two following operations: 
- The series composition, applied upon an arc $u=(x ; y)$, creates a new node $z$ and replaces $u$ by two $\operatorname{arcs} u_{1}=(x ; z)$ and $u_{2}=(z ; y)$ (cf. Figure 1a). We call series the relation that binds $u_{1}$ and $u_{2}$ and denote it $u_{1}+u_{2}$.

- The parallel composition, applied upon an arc $u=(x ; y)$, duplicates $u$ by creating a new one $v=(x ; y)$ (cf. Figure 1b). We call parallel the relation that binds $u$ and $v$ and denote it $u / / v$.

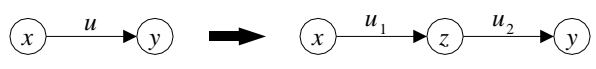

(a)

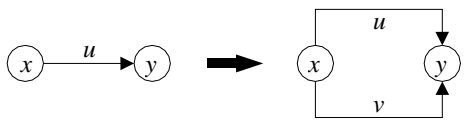

(b)

Figure 1: Series and parallel compositions.

The series and parallel relations are gathered under the term SP-relations. During the construction process, a SP-relation that binds two arcs can become a relation between two series-parallel subgraphs. Hence, the term single SP-relation is introduced to identify a SP-relation between two arcs. From the recursive definition of a SP-graph, it is easy to verify that a SP-graph has always at least a single SP-relation (the SP-relation created from the last composition).

The SP-relations are binary operations, so we can represent a SP-graph by a binary tree called decomposition binary tree or SP-tree (cf. [13], [7]). Figure 2 shows a SP-tree of a SP-graph. Section 3 explains how to find such a tree in linear time.

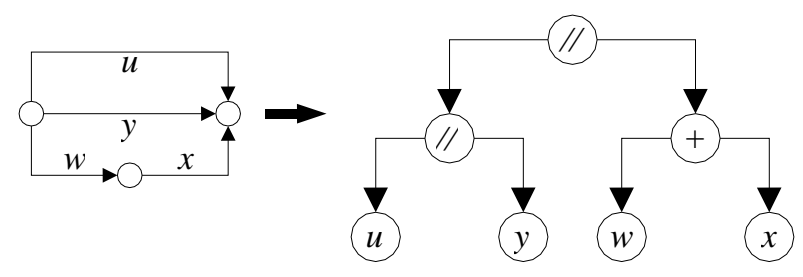

Figure 2: Example of SP-tree.

Numerical results (cf. [4]) showed that linear programming and the out-of-kilter method take advantage of the particular structure of the SP-graphs and behave really better on this class of graphs. However the dual cost-scaling approach does not work that well on these instances, whereas it proves to be the most efficient for non-specific graphs. Moreover, the aggregation method presents the best performance on SP-graphs.

\subsection{Aggregation Method}

The aggregation method, that allows to solve CPLCT only on SP-graphs, has been introduced in [4]. The algorithm works on a SP-tree $T$ of the SP-graph $G$ and is recursive: considering a SPrelation in $T$, it assumes that the optimal tensions of the two subgraphs implied in the relation are known, and from them, it is possible to quickly build the optimal tension of the whole SP-relation. Hence, starting from the leaves of $T$, the optimal tension of each SP-relation is built to finally reach the root of the tree $T$.

From the definition of a SP-graph, it is obvious that a SP-graph has only one source node and only one target node. Hence, the main tension $\bar{\theta}$ of a SP-graph is defined as the tension between its 
source $s$ and target $t$, i.e. $\bar{\theta}=\pi_{t}-\pi_{s}$. To get an efficient algorithm, the minimum cost function $C_{G}$ of a SP-graph $G$ must be defined. This function represents the cost of the optimal tension where the main tension is forced to a given value:

$$
C_{G}(x)=\min \left\{\sum_{u \in U} c_{u}\left(\theta_{u}\right) \mid \theta \in T_{G}, \bar{\theta}=x\right\}
$$

As each function $c_{u}$ is convex, the minimum cost function is indeed convex. Let us consider two series-parallel subgraphs $G_{1}$ and $G_{2}$, and suppose that their minimum cost functions $C_{G_{1}}$ and $C_{G_{2}}$ are known. The minimum cost function $C_{G_{1}+G_{2}}$ of the SP-relation $G_{1}+G_{2}$ is:

$$
C_{G_{1}+G_{2}}(x)=\min _{x=x_{1}+x_{2}} C_{G_{1}}\left(x_{1}\right)+C_{G_{2}}\left(x_{2}\right)
$$

Thus, $C_{G_{1}+G_{2}}$ is the inf-convolution $C_{G_{1}} \square C_{G_{2}}$. It is well known that this operation maintains convexity (e.g. [11]). The minimum cost function $C_{G_{1} / / G_{2}}$ of the SP-relation $G_{1} / / G_{2}$ is:

$$
C_{G_{1} / / G_{2}}(x)=C_{G_{1}}(x)+C_{G_{2}}(x)
$$

Thus, $C_{G_{1} / / G_{2}}$ is simply the sum $C_{G_{1}}+C_{G_{2}}$, which is convex if $C_{G_{1}}$ and $C_{G_{2}}$ are convex. From this assessment, a simple recursive algorithm can be proposed to build the minimum cost function $C_{G}$ of a SP-graph $G$. As explained in [4], it is preferred to deal with the $t$-centered minimum cost function $C_{G}^{t}$ of $G$ that makes the method both more efficient and easier to understand:

$$
C_{G}^{t}(x)=C_{G}(x+t)-C_{G}(t)
$$

This way $C_{G}^{t}$ represents the minimum additional cost to increase or decrease the main tension $\bar{\theta}_{G}$ from a reference tension $t$. If $t=\theta_{G}^{*}$, the minimum cost tension of a graph $G$, the cost function $C_{G}^{*}=C_{G}^{\overline{\theta_{G}^{*}}}$ equals 0 at the optimal tension.

Moreover we are interested in finding the minimum cost tension of $G$, thus the aggregation method provides the minimum cost function $C_{G}^{*}$ with additional information on each of its pieces (cf. [4] for details). It allows for instance to reach optimally a new main tension $t^{\prime}$ from the reference tension $\theta_{G}^{*}$ in linear time (precisely $O(m)$ operations). This facility is widely used in the further reconstruction approach. The whole aggregation method performs in $O\left(\mathrm{~m}^{3}\right)$ operations.

\subsection{Quasi SP-Graphs}

A quasi $S P$-graph or $Q S P$-graph $G=(X ; U)$ is such that the removal of a minimal subset $U^{\prime} \subset U$ of arcs from $G$ makes the remaining graph $G^{\prime}=\left(X ; U \backslash U^{\prime}\right)$ series-parallel. The ratio $\left|U^{\prime}\right| /|U|$ is called the SP-perturbation of the graph $G$. This value indicates how many arcs of $G$ are disturbing the series-parallel property of $G$. From this definition, any connected graph is a QSP-graph, but we prefer to use this term for graphs with a small SP-perturbation (in applications issued from the hypermedia field, $10 \%$ seems a satisfying threshold).

\section{Reconstruction Approach}

As the aggregation method can not be used "as it is" on QSP-graphs and the dual cost scaling approach proves to be less efficient than the out-of-kilter on SP-graphs, we propose in this section a method called reconstruction to solve CPLCT on QSP-graphs. This new approach combines the aggregation and the out-of-kilter techniques based on a SP-decomposition of the graph. 


\subsection{Decomposition Phase}

We call series-parallel component or SP-component of a graph $G$ a SP-subgraph of $G$. A partition $P$ of the arcs of $G$ defines a series-parallel decomposition or $S P$-decomposition of $G$, where each set of arcs in $P$ induces a SP-subgraph of $G$. A SP-decomposition of $G$ is thus a set of arc-disjoint SP-components, whose union is $G$. Section 3 discusses ways to obtain a "good" SP-decomposition that fits the reconstruction process.

The reconstruction method starts with the search of a SP-decomposition $D$ of the graph $G$. Then CPLCT is solved on each SP-component of $D$ with the aggregation method. Thus, for each component $D_{u} \in D$, its minimum cost function $C_{u}^{*}$ and its optimal tension $\theta_{u}^{*}$ are known, so $D_{u}$ can be seen as a single aggregated arc $u$ with a convex piecewise linear cost function $c_{u}=C_{u}^{*}$ and a tension $\theta_{u}=\theta_{u}^{*}$.

\subsection{Reconstruction Phase}

The method attempts next to put the SP-components back together. An iterative process consists in adding one by one the aggregated arcs into a new graph, rebuilding the original graph $G$ back. Starting with $H^{0}=\left(X^{0} ; U^{0}\right)=(\emptyset ; \emptyset)$, at each step an aggregated arc $u=(x ; y)$ is added, i.e. $H^{k}=\left(X^{k-1} \cup\{x ; y\} ; U^{k-1} \cup\{u\}\right)$. The newly added arc $u$ is the only one that may be out-of-kilter.

We remind that the kilter curve is defined from the tension $\theta_{u}$ and the flow $\varphi_{u}$ of the arc $u$, and roughly that $\varphi_{u}$ must equal $c_{u}{ }^{\prime}\left(\theta_{u}\right)$ for the arc to be in-kilter. If all the arcs of $H^{k}$ are in-kilter, its tension $\theta$ is optimal. The idea of the out-of-kilter approach is to bring all the arcs on their kilter curve (e.g. [2]).

The newly added arc $u$ may be out-of-kilter because even if its tension is optimal, its flow must be set to 0 in order to keep the flow conservation constraints on the whole graph $H^{k}$. Fortunately, as this arc is the only one out-of-kilter, repetitive search for a cycle or a cocycle to modify respectively either the flow or the tension of the arc $u$ can be performed in $O((A+B) m)$ operations, where $A$ is the maximum tension and $B$ the maximum flow (i.e. the maximum derivative of its cost function) for the arc $u$. Once the arc is in-kilter, the whole tension on $H^{k}$ is optimal and another aggregated arc $v$ can be added.

\subsection{Tension Adjustment}

But adding an arc into the graph is not that obvious. Consider the aggregated arc $u=(x ; y)$, the optimal tension $\theta_{u}$ of $u$ may not be equal to the difference of potentials $\pi_{y}-\pi_{x}$. The tension of the arc $u$ has to be optimally adjusted to be equal to $\pi_{y}-\pi_{x}$. That can be achieved using the minimum cost function $C_{u}^{*}$ of the arc $u$ to adapt optimally the main tension of the SP-component aggregated behind $u$. As said in Section 1, it can be performed in $O(m)$ operations (cf. [4]).

\subsection{SP-Component Splitting}

For the need of the reconstruction, we assume a partial order on the components of the SPdecomposition $D$, such that if the source node (i.e. without any predecessor) and/or the target node (i.e. without any successor) of a SP-component $D_{u}$ belong to component $D_{v}$, then $D_{v}<D_{u}$. Decomposition methods presented in Section 3 prove that such an order exists for any graph. During the reconstruction phase, the SP-components are added following this partial order.

Another problem arises when adding an aggregated arc $u=(x ; y)$ into the graph $H^{k}$ : maybe the source node $x$ and/or the target node $y$ of $u$ are not present in $H^{k}$, because simply hidden in one 
of the aggregated $\operatorname{arcs} v$ of the graph $H^{k}$. Hence we need to split the SP-component behind such an $\operatorname{arc} v$ to make the node visible. The easiest way is to bring back all the arcs of the SP-component $D_{v}$ into the graph $H^{k}$, (i.e. $H^{k}=H^{k-1} \cup D_{v} \backslash\{v\}$ ).

The drawback of this technique is that the biggest SP-component, added behind a single aggregated arc at the first step, is certainly split at the second step, because nodes will obviously be needed for the secondly added arc. This approach reveals to be extremely inefficient, as most of the arcs will be brought back in the graph at the second step only. Thus, we need a smarter way than splitting totally a SP-component. This is the purpose of Section 4 that proposes a technique to split a SP-component into several pieces, reducing their numbers to the minimum necessary.

\subsection{Conservation of the In-Kilter Property}

After the splitting of an aggregated arc $u=(x ; y)$, the resulting arcs must be in-kilter to keep the whole graph $H^{k}$ optimal. Thus, knowing their tension, it is straightforward to find an interval in which their flow must be: indeed, with our assumptions, the kilter curve is a step function (cf. Figure 3).

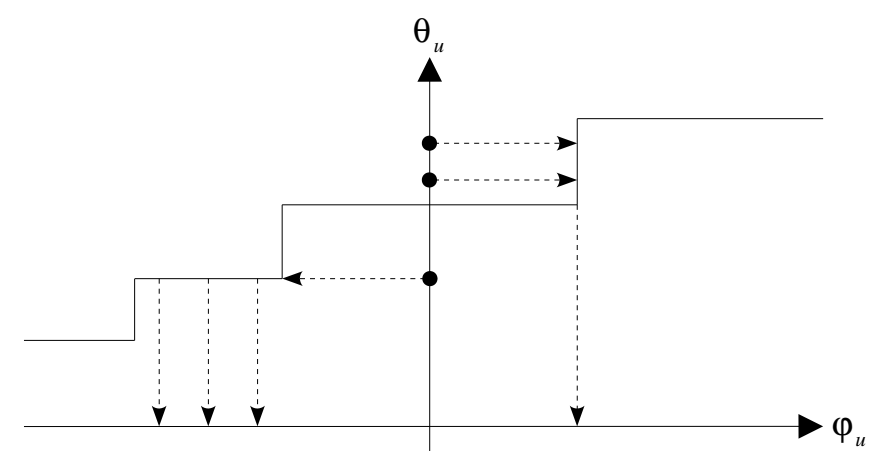

Figure 3: Kilter flow building.

For each arc $v$ of the SP-component $D_{u}$, an interval $\left[e_{v} ; f_{v}\right]$ can thus be defined. Let suppose an arc $w$ from the source node $x$ to the target node $y$ of the SP-component with a capacity $\left[\varphi_{u} ; \varphi_{u}\right]$, where $\varphi_{u}$ is the flow of $u$ in the whole graph $H^{k}$. Find a flow $\varphi_{v}$ for each arc $v$ of the SPcomponent $D_{u}$ turns to find a feasible flow $\varphi$ in the SP-component (with the added loop arc $w)$. We propose to solve it in $O\left(\mathrm{~m}^{3}\right)$ operations with a technique using the SP-tree of the SPcomponent like the aggregation (cf. [3]). However, as this phase is not critical in terms of time consumption, any well-known method is suitable to solve this flow problem.

\subsection{Complexity}

Algorithm 1 summarizes the whole process of the reconstruction method. Let $k=|D|$ and $p_{u}$ be the number of arcs of a SP-component $D_{u}$. The reconstruction algorithm needs, for each SPcomponent of $D$, eventually a splitting (with the tension adjustment and the conservation of the in-kilter property), an aggregation $\left(O\left(p_{u}^{3}\right)\right.$ operations) and an out-of-kilter iteration $(O(m(A+B))$ operations). The whole splitting phase need $O\left(\min \{n ; 2 k\} m^{3}\right)$ operations (cf. Section 4). Thus, the reconstruction method requires $O\left(\min \{n ; 2 k\} m^{3}+k m(A+B)\right)$ operations. 


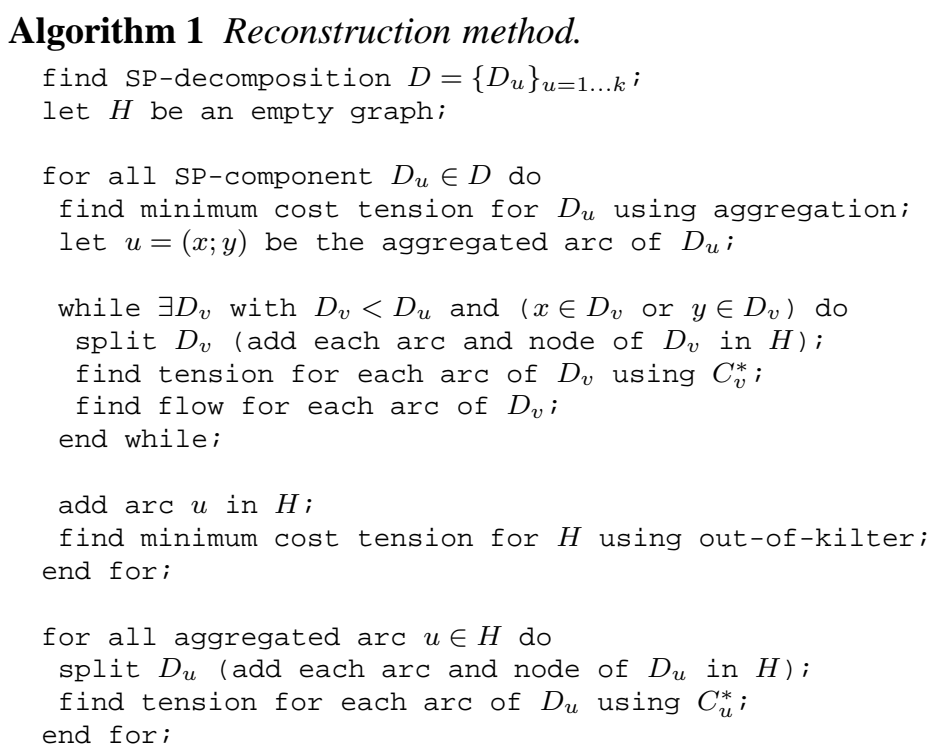

\section{Series-Parallel Decomposition}

The recognition of a SP-graph is known for long as an easy problem that can be solved in linear time (cf. [13]). The various algorithms proposed by many authors can immediately be adapted and without altering their complexity to build the SP-tree during the recognition phase. These methods being very efficient, our discussion will not be on their complexity, but on the way they recognize a SP-graph, our goal being to find for any graph the "best" SP-decomposition suitable for the reconstruction process.

The characterization of such a decomposition is not that obvious. If it is too compact, i.e. with few SP-components, that will tend to produce a lot of splittings during the reconstruction (like the phenomenon explained for the first SP-component in Section 2). At the opposite if the decomposition is scattered, i.e. with many SP-components, the aggregation phase will become useless and the reconstruction will tend to a single out-of-kilter algorithm.

We choose to study here two recognition methods, the reduction and the path approaches, and propose heuristic extensions to find a "good" SP-decomposition. To present these algorithms, a recursive notation of the SP-trees is introduced. A tree with a root $a$, a left subtree $T_{l}$ and a right subtree $T_{r}$ is represented by $\left(a ; T_{l} ; T_{r}\right)$. The reverse operations of the series and parallel compositions, called $S P$-reductions, are defined as follows:

- The series reduction, noted $S_{x}^{-1}$, replaces the SP-relation $(y ; x)+(x ; z)$ by an arc $(y ; z)$.

- The parallel reduction, noted $P_{u}^{-1}$, replaces the SP-relation $u / / v$ by the arc $u$.

\subsection{Reduction Approach, Recognition}

This recognition approach is widespread because very intuitive. As explained in Section 1, a SPgraph has at least one single SP-relation. The idea is to find one, apply the associated SP-reduction and repeat until the graph is reduced to a single arc. If there is no more single SP-relation and the graph is not a single arc, that means the graph is not series-parallel.

This method has been proposed first in [13]. It appears then in [12] with some improvement: all the multiple arcs are first removed (no single parallel relation exist anymore), then series and 
parallel-and-series (composition of a parallel relation with a series relation) relations are detected and reduced. The detection is easier (only the nodes are checked) and the reduction is more efficient (because with the parallel-and-series relation, two arcs and one node are removed in one step). However, this improvement does not change fundamentally the way the algorithm performs. A similar variant is proposed in [5] which proposes 18 reductions, most of them working only on non-directed graphs. These improvements are difficult to take into account with our goal of finding a SP-decomposition, because our graphs have non-specific structures, so the occurrences of these special reductions dedicated to SP-graphs will be rare.

\subsection{Reduction Approach, Decomposition}

A generic version of the method is presented in Algorithm 2. It builds at the same time a SPdecomposition of the graph where each SP-component is represented by its SP-tree. For this purpose, a function $t$ is defined that associates a SP-tree $t_{u}$ with each arc $u$ of the graph $G$. At the beginning, each arc possesses a SP-tree with a single node that is the arc itself. We sketch below what happens during a SP-reduction.

- The series reduction $S_{x}^{-1}$ removes the two $\operatorname{arcs} u=(y ; x)$ and $v=(x ; z)$ of the relation $u+v$, and creates an arc $w=(y ; z)$. The SP-tree of $w$ is then $\left(+; t_{u} ; t_{v}\right)$, the old $t_{u}$ and $t_{v}$ are removed.

- The parallel reduction $P_{u}^{-1}$ removes the arc $v$ of the relation $u / / v$. The SP-tree of $u$ becomes $\left(/ / ; t_{u} ; t_{v}\right)$, the old $t_{u}$ and $t_{v}$ are removed.

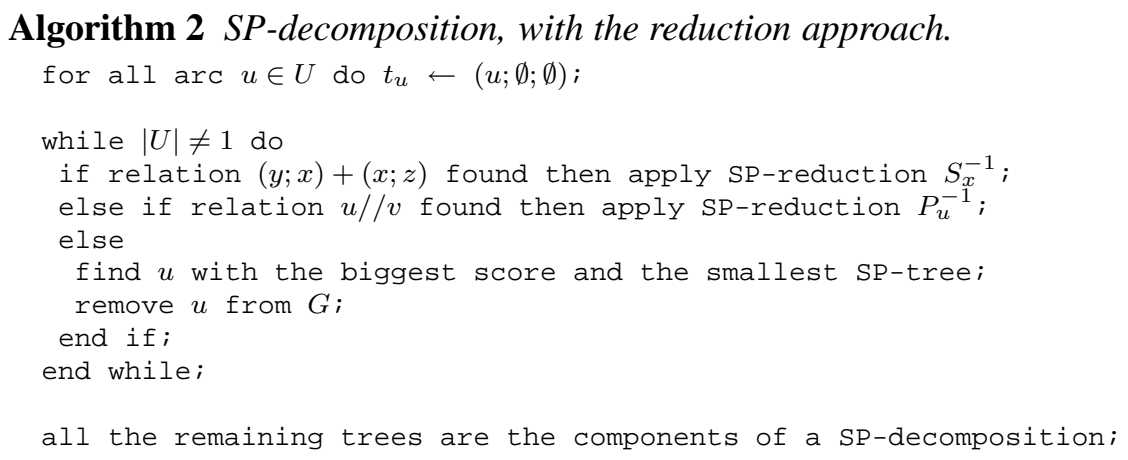

To recognize a SP-graph, the method reduces successively each single SP-relation until there is either only one arc in the graph or no more single SP-relation to reduce. To find a whole SPdecomposition of any graph, the algorithm repeats until it blocks, so an arc must be removed to reveal SP-relations and allow to continue the reductions. When suppressing an arc, no parallel relation can appear, only series relations can. So the intuitive idea, whenever a blocking occur, is to remove the arc that reveals the most series relations, and if none exist the one that will contribute in revealing the most series relations. We propose a heuristic approach that assigns two scores to each node $x$ : one used when $x$ is source of an $\operatorname{arc}\left(s_{x}^{+}\right)$and another used when $x$ is the target of an $\operatorname{arc}\left(s_{x}^{-}\right)$. The score of an $\operatorname{arc} u=(x ; y)$ is $s_{x}^{+}+s_{y}^{-}$, and is calculated as follows:

$$
\begin{aligned}
& s_{x}^{+}=\left\{\begin{aligned}
-M, & \text { if } d_{x}^{+}=1 \\
0, & \text { if } d_{x}^{+}>1 \text { and } d_{x}^{-}=0 \\
1 /\left(d_{x}^{+}-1\right)+1 / d_{x}^{-}, & \text {if } d_{x}^{+}>1 \text { and } d_{x}^{-}>0
\end{aligned}\right. \\
& s_{x}^{-}=\left\{\begin{aligned}
-M, & \text { if } d_{x}^{-}=1 \\
0, & \text { if } d_{x}^{-}>1 \text { and } d_{x}^{+}=0 \\
1 /\left(d_{x}^{-}-1\right)+1 / d_{x}^{+}, & \text {if } d_{x}^{-}>1 \text { and } d_{x}^{+}>0
\end{aligned}\right.
\end{aligned}
$$

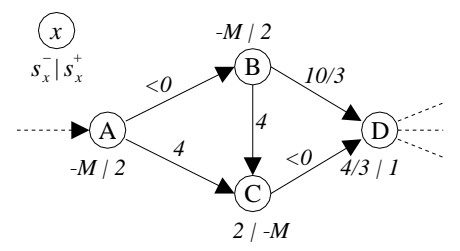

Figure 4: Example of scoring. 
Here $d_{x}^{+}$represents the number of arcs outgoing $x$ and $d_{x}^{-}$the number of arcs incoming $x$. Intuitively, a node $x$ with $d_{x}^{+}=2$ and $d_{x}^{-}=1$ is the best candidate to be the source of the arc to remove because it reveals a series relation, its score is 2 . The same way, an arc $y$ with $d_{y}^{-}=2$ and $d_{y}^{+}=1$ is the best candidate to be the target of the arc to remove. The best candidate arc has a score of 4 . This score tends to decrease first for the extremities of the arcs having important numbers of incoming and outgoing arcs, because that means numerous arc suppressions are needed before they can become series relations. Removals that alter the connectivity of the graph are penalized with a negative score, hence if a node would have no incoming or outgoing arc after the suppression, its score is set to $-M$, with $M$ such that even the best score for the other extremity will not make the score of the arc positive, any $M>2$ is suitable. Figure 4 shows an example of scoring. In the case of scores equality, the arc that hides the smallest SP-component is removed, with the hope of revealing finally the biggest SP-component. If we consider no specific data structure for the graph, the complexity of Algorithm 2 is $O\left(\mathrm{~m}^{2}\right)$ operations, at each step the selection of the arc to be removed requires $O(m)$ operations (to assign a score to each remaining arc of the graph).

\subsection{Path Approach, Recognition}

The paths of a SP-graph are organized in a very specific way. In [9], this is formalized by the concept of ear decomposition. We propose here a quite different approach based on two kinds of nodes: the branching nodes (with more than one outgoing arc) and the synchronization nodes (with more than one incoming arc).

In a SP-graph, such nodes represent respectively the beginning and the end of parallel relations. We call branching two arcs outgoing a branching node (i.e. the beginning of a parallel relation). In the same way, a synchronization represents two arcs incoming a synchronization node (i.e. the end of a parallel relation). By mean of clarity, we sometimes identify a branching (respectively synchronization) by its branching (respectively synchronization) node.

The approach proposed here is based on a search through the graph in the topological order of the nodes (i.e. a node is visited only after all its predecessors). Let $S_{k}$ be the set of nodes marked during the process until iteration $k$. A branching will be said closed at iteration $k$ if there is a synchronization node $y \in S_{k}$ such that two arc-distinct directed paths $P_{1}$ and $P_{2}$ between $x$ and $y$ exist and contain each one of the two arcs of the branching; the two arcs incoming $y$ that belong each to one of the paths form a synchronization $y$ that closes $x$. A closed branching is associated with the first synchronization encountered during the search that closes it. The paths that allow the closure of the branching (in our definition $P_{1}$ and $P_{2}$ ) are called the closure paths of the branching.

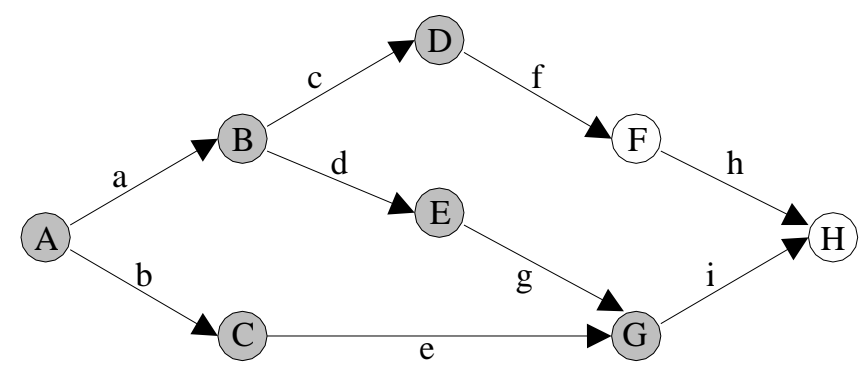

Figure 5: Example of branchings.

To illustrate these definitions, consider Figure 5. The gray nodes represent visited nodes at iteration $k$. $A$ and $B$ are branching nodes, $G$ and $H$ are synchronization nodes. $(a ; b)$ is a closed 
branching, the associated synchronization is $(e ; g)$. On the contrary, branching $(c ; d)$ is open. Closure paths for branching $(a ; b)$ are $(a ; d ; g)$ and $(b ; e)$. The graph of the example is not seriesparallel. Intuitively, the problem comes from the open branching $(c ; d)$. Let us prove the following proposition.

Proposition 1 A graph is series-parallel if and only if at each iteration of the topological search through the graph, for each closed branching $x$, there are two closure paths between the branching $x$ and its associated synchronization $y$ that do not possess any open branching.

Proof: $(\Rightarrow)$ At a given iteration, suppose there is an open branching $z$ on one of the closure paths of a closed branching $x$ associated with its synchronization $y$. That gives a graph with the general aspect presented by Figure 6a. Attempting a reduction with Algorithm 2 (with no arc suppression), the best that can be found is the graph illustrated by Figure $6 \mathrm{~b}$. The reduction can thus not be terminated without any arc suppression, so the graph is not series-parallel.

$(\Leftarrow)$ Suppose now that no such open branching exist, the recognition Algorithm 3 presented further proves that it is possible to find (with no arc suppression) the SP-tree associated with the graph, so it is series-parallel.

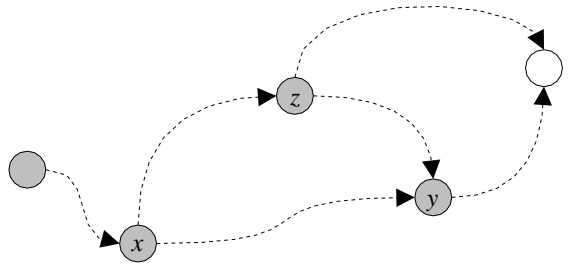

(a) Open branching

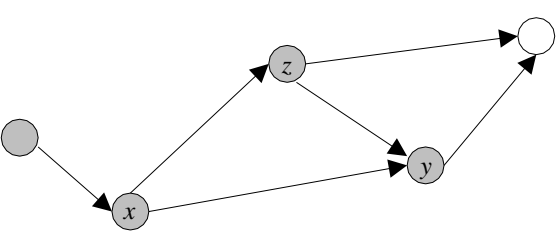

(b) Graph after SP-reduction

Figure 6: Example of open branching on closure path.

To verify the proposition (in order to determine if a graph is series-parallel), we propose a marking process that allows to identify, arriving on a synchronization, the branchings that are closing and if a branching is open in one of the closure paths. Let $\Delta_{u}$ be the signature of an arc $u, \Delta_{u}=x$ means $x$ is the last (according to the topological order of the nodes) open branching between the source node of the graph and the arc $u$. Let $\Delta_{x}$ be the signature of a node $x$, it is a couple $\Delta_{x}=\left(l_{x} ; d_{x}\right)$. If the node is not a branching node then $d_{x}=0$, else $d_{x}$ indicates the number of branchings located at node $x$ that are open. $l_{x}$ indicates the level of branching, e.g. if $l_{x}=2$ then there are two open branchings (including $x$ ) in the paths between the source node of the graph and the node $x$.

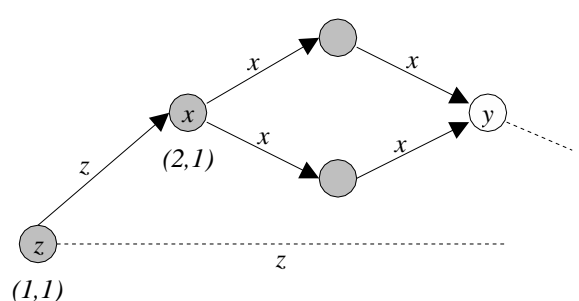

(a) Iteration $k$

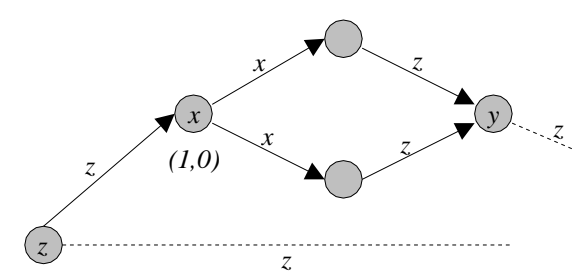

(b) Iteration $k+1$

Figure 7: Example of signature change.

The signature of an arc or a node can change while searching the graph in the topological order of the nodes. When two arcs $u$ and $v$ with the same signature $x$ (at iteration $k$ ) meet at a 
synchronization node $y$ (at iteration $k+1$ ), they form a synchronization and closes thus a branching of node $x$ (cf. Figure 7a). The signature of their branching is then modified: $d_{x}$ is decreased of 1. If $d_{x}$ equals 0 , that means all the branchings of node $x$ are closed. The signature of the arcs $u$ and $v$ must change, it becomes the open branching before $x$, i.e. the signature of one of the incoming arcs of branching $x$ (cf. Figure 7b). Ideally, the signature of all the arcs of signature $x$ must change, but for the needs of the algorithm (that searches the graph in the topological order of the nodes and thus never come back on already used arcs) this modification is not necessary.

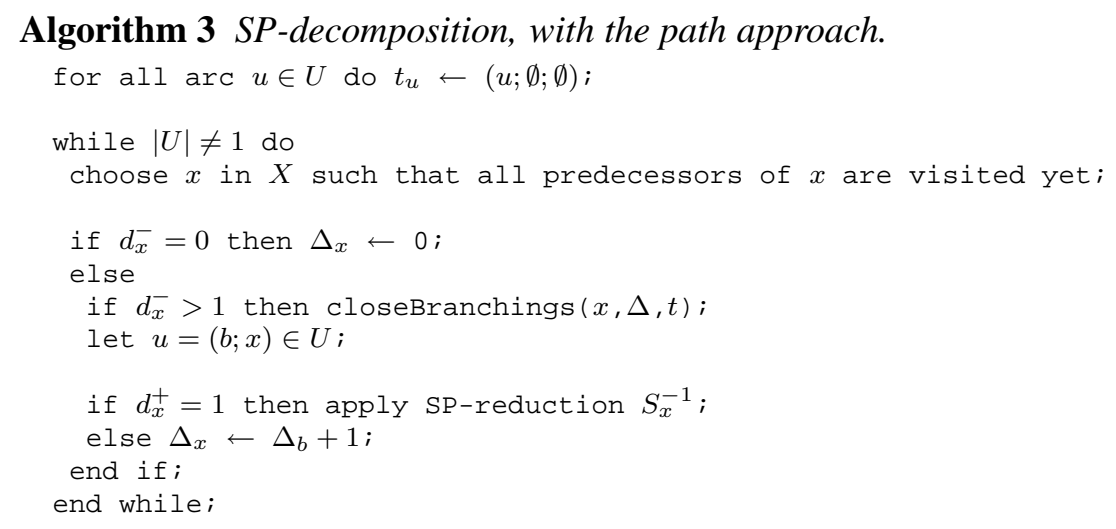

Algorithm 3 proposes to search the graph following the topological order of the nodes, marking progressively the nodes and the arcs with the signature $\Delta$. It is supposed that the graph has only one source and at least one arc, else it is sure that it is not series-parallel. From the source of the graph, the algorithm marks the nodes and the arcs of the graph. At each synchronization node $y$, it modifies certain signatures to represent the closure of associated branchings (cf. Algorithm 4). If at the end of this step, two incoming $\operatorname{arcs} u_{1}$ and $u_{2}$ are not marked with the same signature, that means the graph is not series-parallel, because there are two paths $P_{1}$ and $P_{2}$ between the source and respectively $u_{1}$ and $u_{2}$. These paths have at least one branching node in common (at least the source). Let $x$ be the last node (in the topological order) verifying the condition. If there were no open branching on the closure paths between $x$ and $y, u_{1}$ and $u_{2}$ should have the same signature $x$. If they do not, that means at least one of the closure paths between $x$ and $y$ has an open branching, which signature is carried by $u_{1}$ or $u_{2}$.

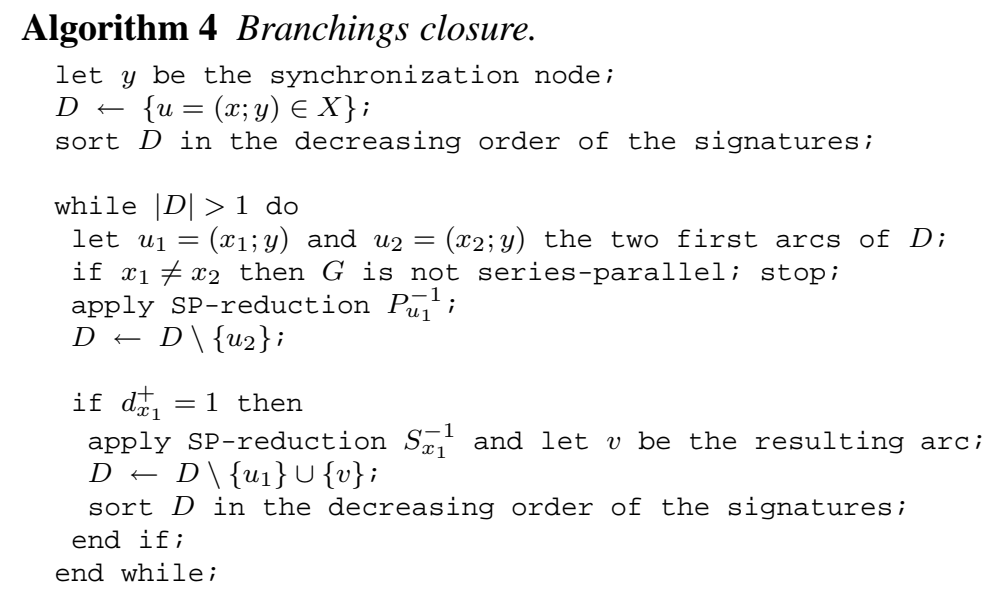

Algorithm 4 performs the closure of the branchings at a given synchronization node $y$, and modifies consequently the signatures. It must check two-by-two the arcs incoming $y$ and if they have identical signatures, close the corresponding branching if not already done. For an efficient search among the arcs, we propose to sort them in a set $D$, in the decreasing order of their signatures, i.e. $u$ before $v \Rightarrow l_{\Delta_{u}}>l_{\Delta_{v}}$ or $\left(l_{\Delta_{u}}=l_{\Delta_{v}}\right.$ and $\left.\Delta_{u}>\Delta_{v}\right)$ (we suppose any order on the 
nodes, the idea is that arcs with the same signature are side-by-side). Hence, when looking the first two arcs of $D$, the priority is given to the branching with the highest level, which must absolutely be closed before any lower level branching. This process requires $O(m \log m)$ operations. So the whole Algorithm 3 requires $O(n m \log m)$ operations.

Algorithm 3 can easily be adapted, without altering its complexity, to build also the SP-tree associated with the graph, as in the reduction approach. The idea consists in reducing the graph with a series reduction each time a node with only one incoming and one outgoing arcs is visited. When a branching is closed, that means a parallel operation has been detected, it must then be reduced. The SP-tree is built once the whole graph is visited (there will remain then only a single arc). The management of the signatures of the arcs and the nodes is then facilitated, because the SP-reductions imply automatically the signatures. For instance, the signature $\Delta_{x}$ of a node $x$ is limited to $l_{x}$ (because $d_{x}$ is always equal to $d_{x}^{+}-1$ ), and the signature of an arc $u=(x ; y)$ is not necessary anymore, because it is always equal to $x$.

\subsection{Path Approach, Decomposition}

This approach offers a new way of recognition of a SP-graph, based on a single search through the graph in the topological order of the nodes. We explain now how to modify this method to build a SP-decomposition of the graph. With this algorithm, there are two ways to discover that a graph is not series-parallel. First, during the search through the nodes according to their topological order, if a circuit exists, the process loops (in Algorithm 3). But the circuit contains one of the nodes visited at last (i.e. nodes for which the successors have not been all visited yet), let $S$ be the set of these nodes. In fact, no more nodes can be visited because two nodes at least of $S$ are predecessors of each other, directly or indirectly. The process will be then to find such a circuit by looking one by one the incoming arcs of each node of $S$. Once the circuit is found, the implied incoming arc is removed and the recognition algorithm can go on.

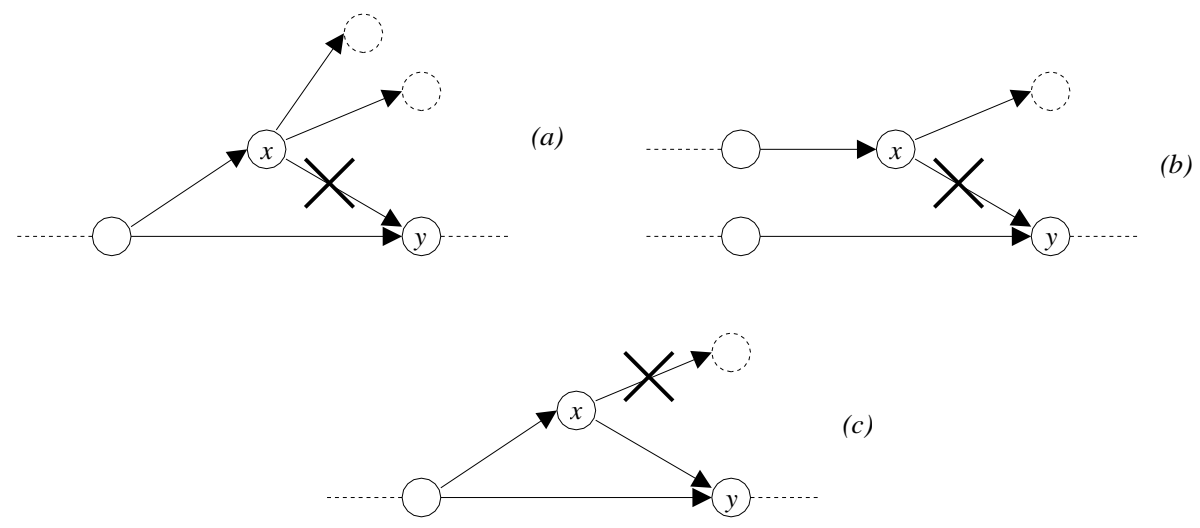

Figure 8: Examples of cases for arc deletion.

Another way to find that a graph is not series-parallel is when it is impossible to establish a synchronization $y$, i.e. an incoming $\operatorname{arc} u=(x ; y)$ has a signature different of the signatures of all the other arcs incoming $y$. Two intuitive possibilities remain then: either remove this arc (cf. Figure 8a) or if $x$ has precisely two outgoing arcs ( $u$ and another $v$ ) as in Figures $8 \mathrm{~b}$ and $8 \mathrm{c}$, verify that the suppression of $v$ can not offer to $u$ the same signature than one of the other incoming arcs of $y$. Thus, we check that the signature of the incoming arc of $x$ is identical with at least one of the signatures of the incoming arcs of $y$. To sum up, when an arc $u=(x ; y)$ blocks the recognition, either it is removed, or its neighbor at branching $x$ is removed, which allows the synchronization of $u$ with one of its neighbor at synchronization $y$. These modifications to build 
the SP-decomposition of the graph does not alter the complexity of the recognition Algorithm 3, because it is only punctual checks at the signatures of nodes.

\section{SP-Component Splitting}

\subsection{Reconstruction Method Extension}

As explained in Section 2.4, the reconstruction approach adds one by one the aggregated arcs in the graph $H^{k}$. However, when adding a new arc $u=(x ; y), x$ and/or $y$ may not exist, because hidden behind an aggregated arc of $H^{k}$. The node $x$ is said inside the component $D_{v}=\left(X_{v} ; U_{v}\right)$ if and only if $x \in X_{v}$ and is neither the source nor the target of $D_{v}$.

Suppose now that $x$ is inside the SP-component $D_{v}$. The first idea in Section 2.4 was to remove the aggregated arc $v$ from $H^{k}$ and replace it by the whole component $D_{v}$ (i.e. all the $\operatorname{arcs}$ of $D_{v}$ are added into $H^{k}$, this way $x$ is not inside a component anymore), but this approach is not making the reconstruction very efficient as it does not benefits really from the aggregation.

The second idea we present here is to minimally split the component $D_{v}$, i.e. find a SPdecomposition $E_{v}$ of $D_{v}$ so $x$ is not inside a SP-component anymore with $\left|E_{v}\right|$ minimal, in order to preserve aggregated arcs as long as possible during the reconstruction process. In this case, $D_{v}$ will be replaced in $H^{k}$ by $E_{v}$ where each component of $E_{v}$ will be represented by an aggregated arc.

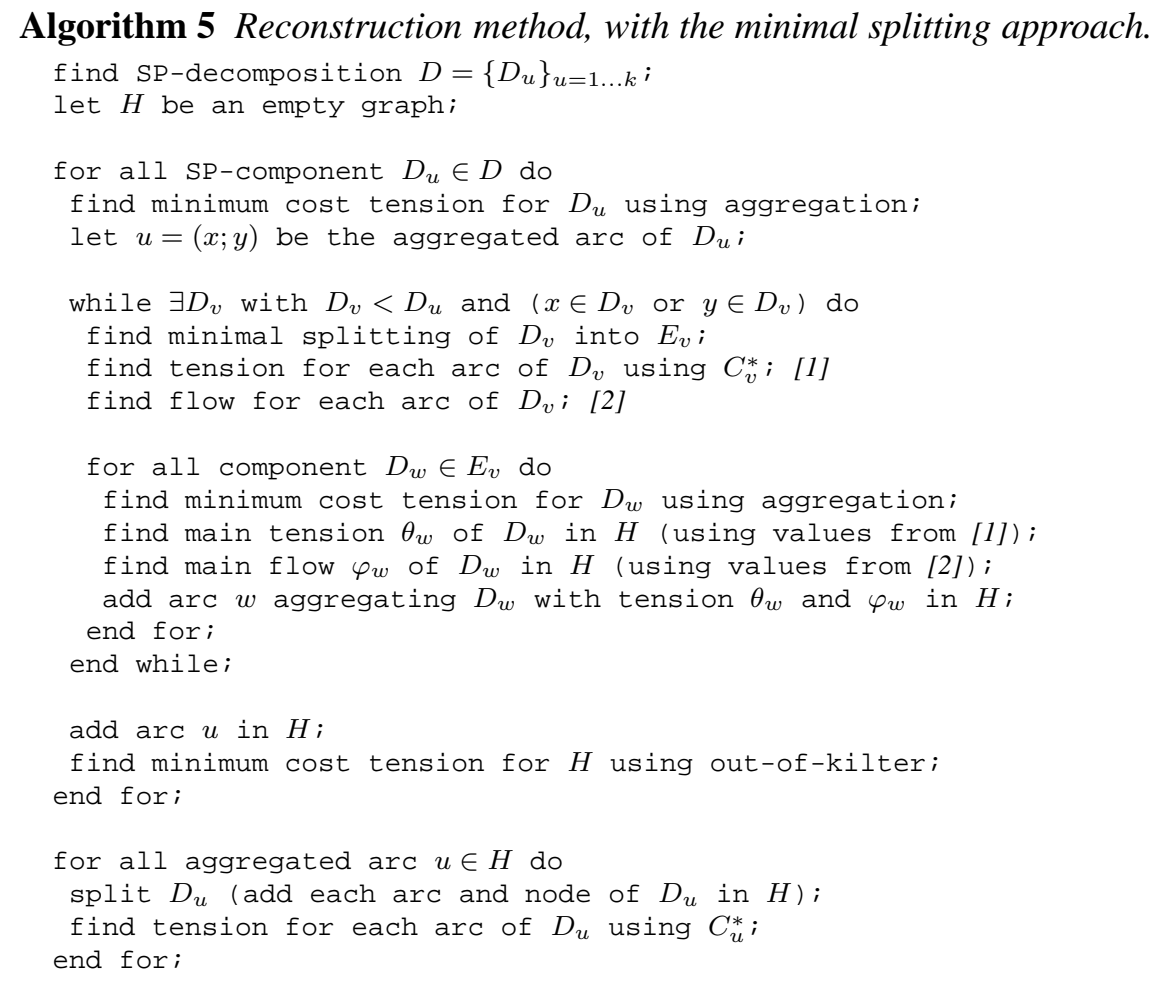

Algorithm 5 proposes modifications for the reconstruction Algorithm 1 of Section 2, in order to consider a minimal splitting of the SP-components instead of their whole splitting. When a component is split, the flow and tension of all its arcs are still computed. And for each SPcomponent $D_{w}$ of the splitting, its cost function $C_{w}^{*}$ is determined using the aggregation method. To insert the arc $w$ aggregating $D_{w}$ into the graph $H^{k}, w$ must have a valid flow and tension. They are computed directly from the values (worked out from [1] and [2] in Algorithm 5) of the single arcs in the component $D_{w}$. 


\subsection{Minimal Splitting}

This section presents a technique to perform the minimal splitting of a SP-component. First, we can notice that $x$ will always originate from a series composition, simply because parallel compositions never create a new node. (1) The first step will be to identify in the SP-tree $T_{v}$ associated with the SP-component $D_{v}$ which series composition generates $x$. (2) Then the two subgraphs of this series composition are extracted from $D_{v}$, they form thus the two first SP-components of $E_{v}$. However by the way they have been extracted, their source and target nodes (except $x$ ) stay inside $D_{v}$, so the problem has been moved from $x$ to two other nodes $z_{1}$ and $z_{2}$. (3) The last step consists thus in going up the SP-tree until the root, and split some of the series compositions so that $z_{1}$ and $z_{2}$ (and later the nodes these new splittings will generate) so they are not inside any SP-component.

\subsubsection{Phase 1: Splitting Pivot Search}

The series composition that generates $x$ must be identified. The SP-tree is explored from its root to its leaves, taking care of memorizing (using for instance a stack) the path that leads us to an arc $a$ that has $x$ as source or target. Once this arc is identified, we follow the path back to the root of the SP-tree, but during this trip we will try to find which series composition generates $x$. Supposing $x$ inside $D_{v}$, the idea of Algorithm 6 is that if $x$ is the source of $a$, then $a$ is part of the right member of a series composition. On the opposite, if $x$ is the target of $a$, then $a$ is part of the left member of a series composition. Algorithm 6 results with $p$ as the pivot of the splitting, i.e. the SP-tree of the series composition that generates $x$.

Algorithm 6 Splitting pivot search.
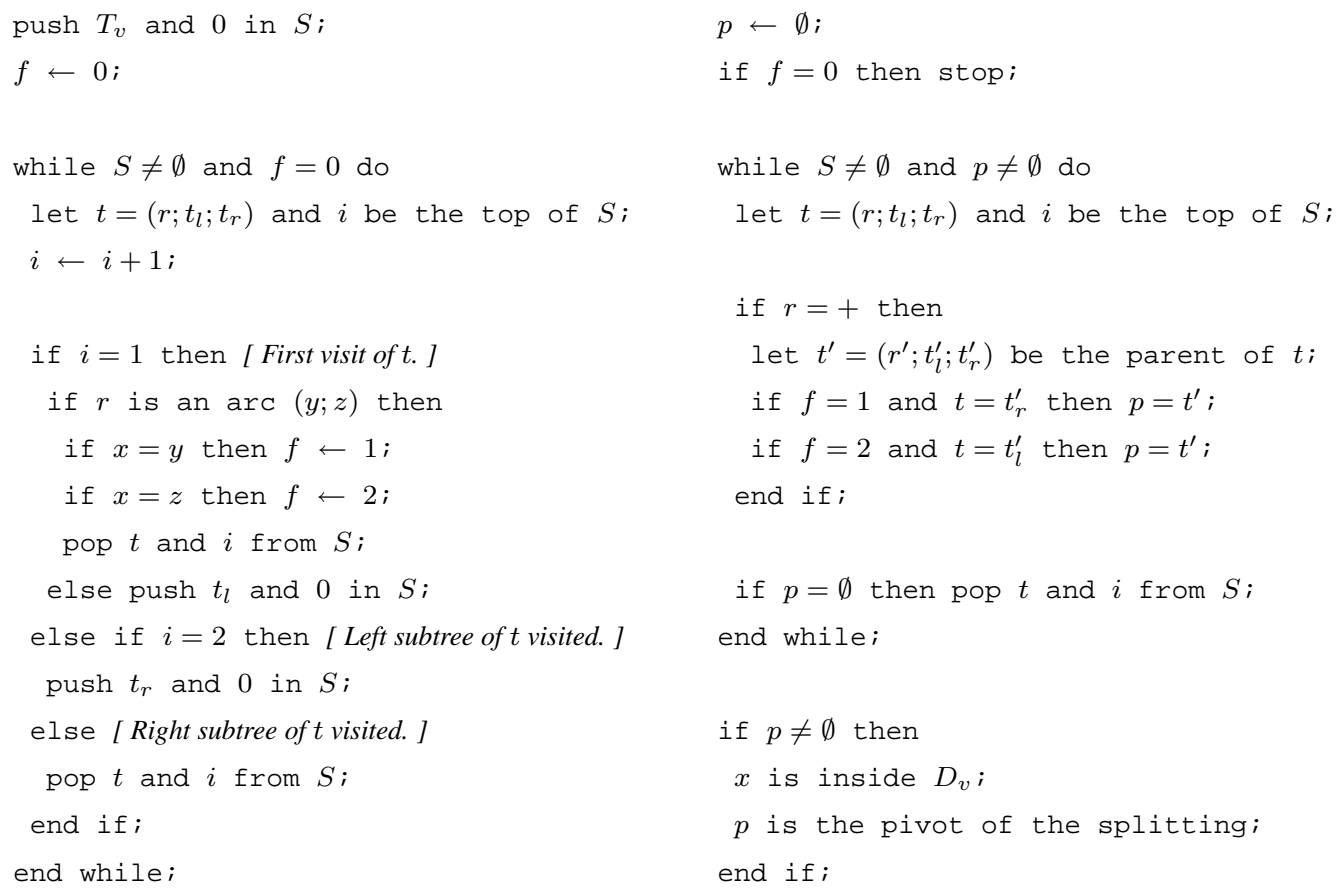

\subsubsection{Phase 2: First Splitting}

Now the pivot is identified, the SP-tree $T_{v}$ has to be split. We can extract $p_{l}$ and $p_{r}$, the subtrees of $p$, from $T_{v}$. If the parent of $p$ is a parallel composition or if $p$ is the root of $T_{v}, T_{v}$ remains a 
SP-component after the extraction. But if the parent of $p$ is a series composition, $T_{v}$ will not be a SP-tree anymore.

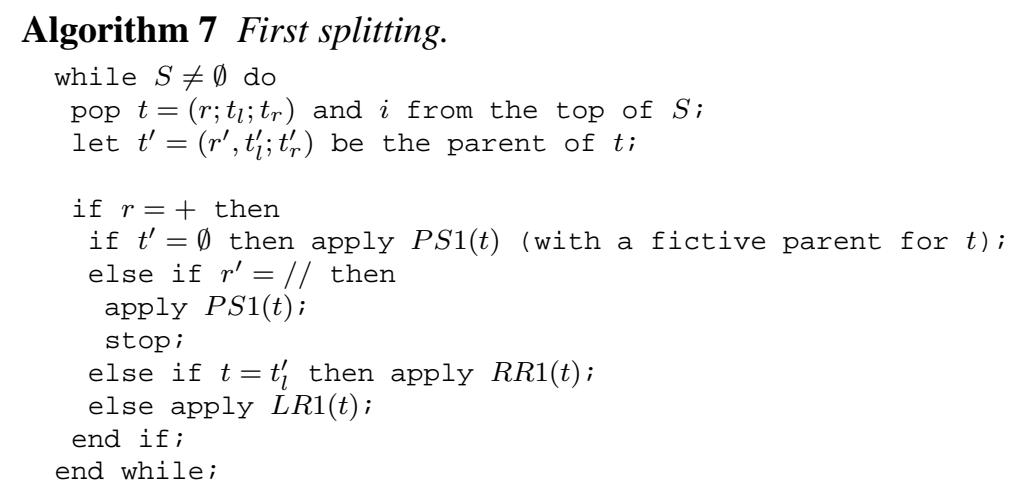

The idea of the second phase is to go on following the path up to the root of $T_{v}$, while neither a parallel composition, nor the root of $T$ is found. Once it happens, the last series composition $t$ encountered is split by the PS1 ("Parallel Splitting I") operation illustrated by Figure 9, its two subtrees $b$ and $c$ being the first components of $E_{v}$.

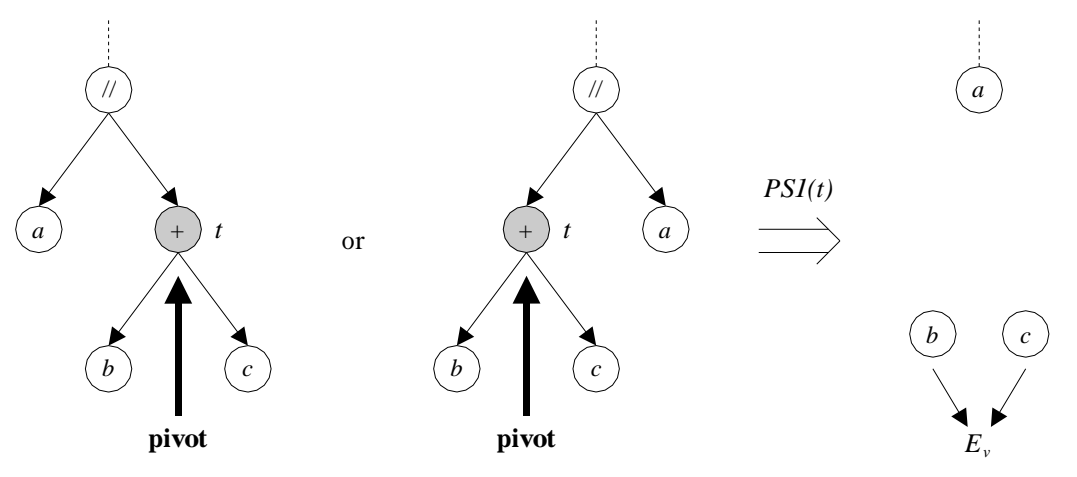

Figure 9: Parallel Splitting I.

But $t$ is not necessary the pivot of the splitting for $x$, hence on the path up to the root of $T_{v}$, for each series composition, rotations must be performed to maintain the pivot as the current series composition. Algorithm 7 uses, depending on the situation, the operation LR1 ("Left Rotation 1", cf. Figure 10) or the operation RR1 ("Right Rotation I", cf. Figure 11) to perform the rotations.

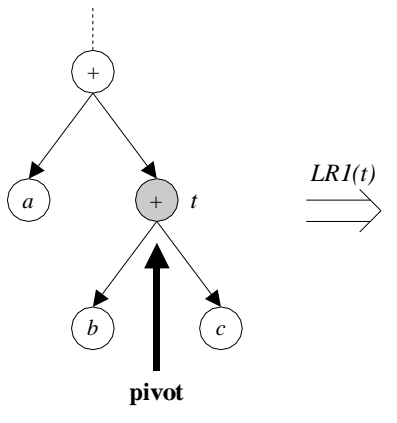

Figure 10: Left Rotation I.
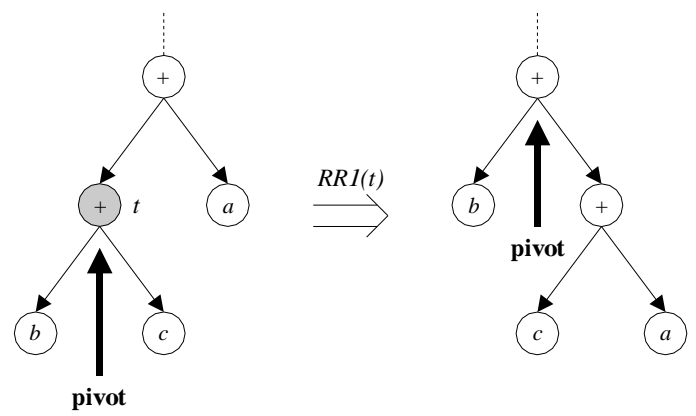


\subsubsection{Phase 3: Second Splitting}

During $P S 1(t)$, two subtrees $b$ and $c$ are extracted from $T_{v} . x$ is no more inside $T_{v}$, because it is target of $b$ and source of $c$. If $t$ is the root of the SP-tree $T_{v}$, then the splitting is over, but on the other cases, the source node $z_{1}$ of $b$ and the target node $z_{2}$ of $c$ are inside the remains of $T_{v}$. To get them out, the parallel peer $a$ of the subtree $b+c$ (cf. Figure 9) has to be extracted too, depending on the situation, with the operation LS ("Left Splitting", cf. Figure 12) or the operation $R S$ ("Right Splitting", cf. Figure 13).

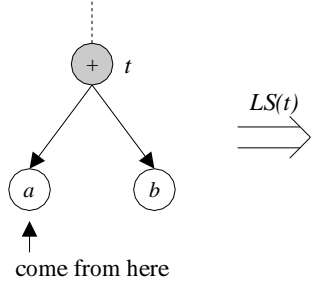

Figure 12: Left Splitting.

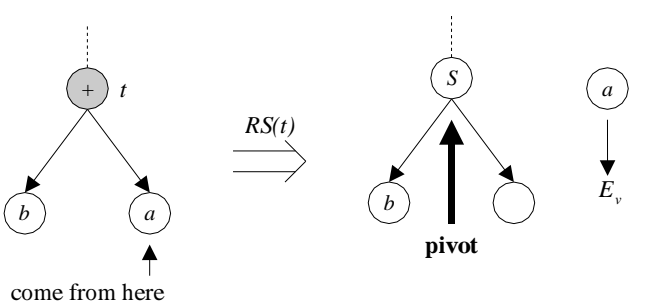

Figure 13: Right Splitting.

Now there is a "hole" in the SP-tree. But this hole is not different of the one made if $x$ were removed in phase (2). So like we maintained the current series composition $t$ as pivot of $x$ along the path back to the root of $T_{v}$ in phase (2), Algorithm 8 maintains the current series composition $t$ as pivot of the "hole". Depending on the situation, operation LR2 ("Left Rotation II", cf. Figure 14) or operation RR2 ("Right Rotation II", cf. Figure 15) are used to perform rotations in the SP-tree $T_{v}$.

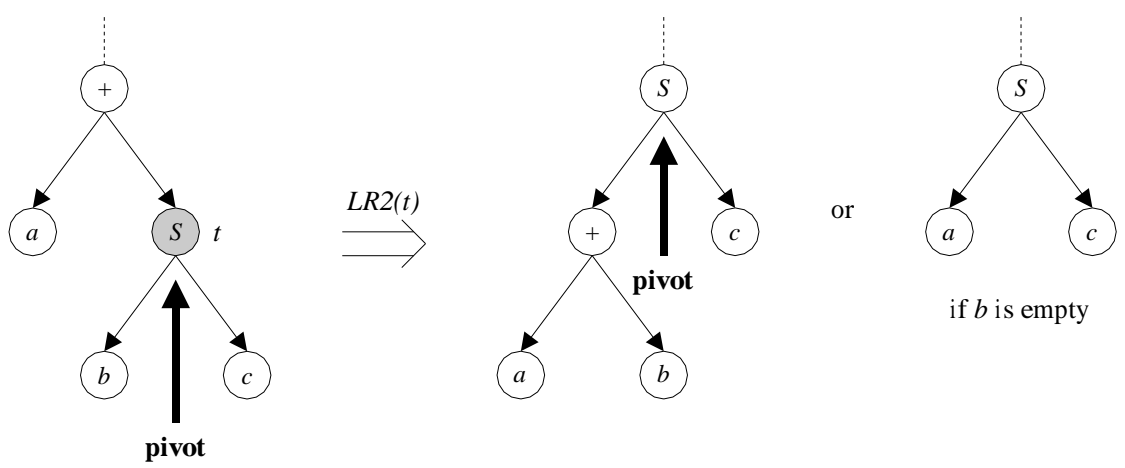

Figure 14: Left Rotation II.

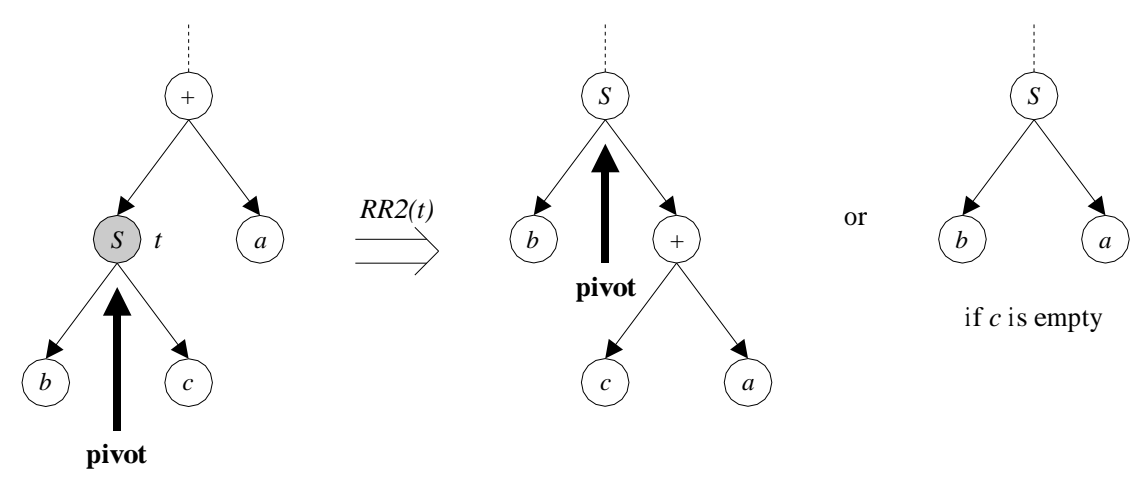

Figure 15: Right Rotation II. 
As in phase (1), once a parallel composition is found, the subtrees $b$ and $c$ of the last series composition $t$ encountered on the path back up to the root of $T_{v}$ are extracted from $T_{v}$ to become SP-components of $E_{v}$, using the operation PS2 ("Parallel Splitting II", cf. Figure 16). Once this splitting achieved, the "hole" problem remains, hence the process of phase (3), cf. Algorithm 8, loops until the root of $T_{v}$ is reached. The operations $L R 2, R R 2$ and $P S 2$ of phase (3) are quite different from the operations $L R 1, R R 1$ and $P S 1$ of phase (2), only because one of the subtrees ( $b$ or $c$ ) of the current series composition $t$ can be empty (due to the splitting from $L S$ or $R S$ at the beginning of the phase).

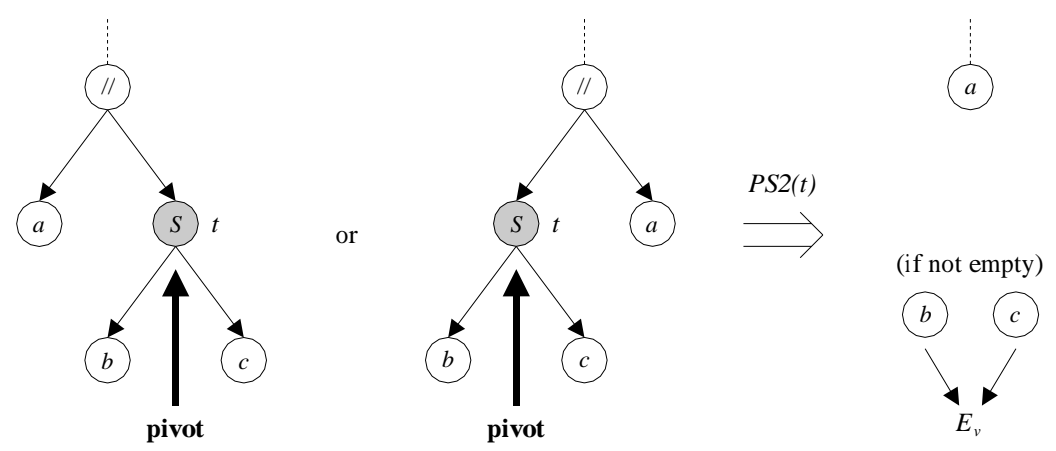

Figure 16: Parallel Splitting II.

The whole splitting process, regrouping the three phases, only needs $O(m)$ operations, the worst it can do is to visit all the nodes of the SP-tree $T_{v}$, which has $2 m-1$ nodes ( $m-1$ for the SP-relations and $m$ for the arcs). All the operations $L R 1, L R 2 \ldots$ need $O(1)$ operations.

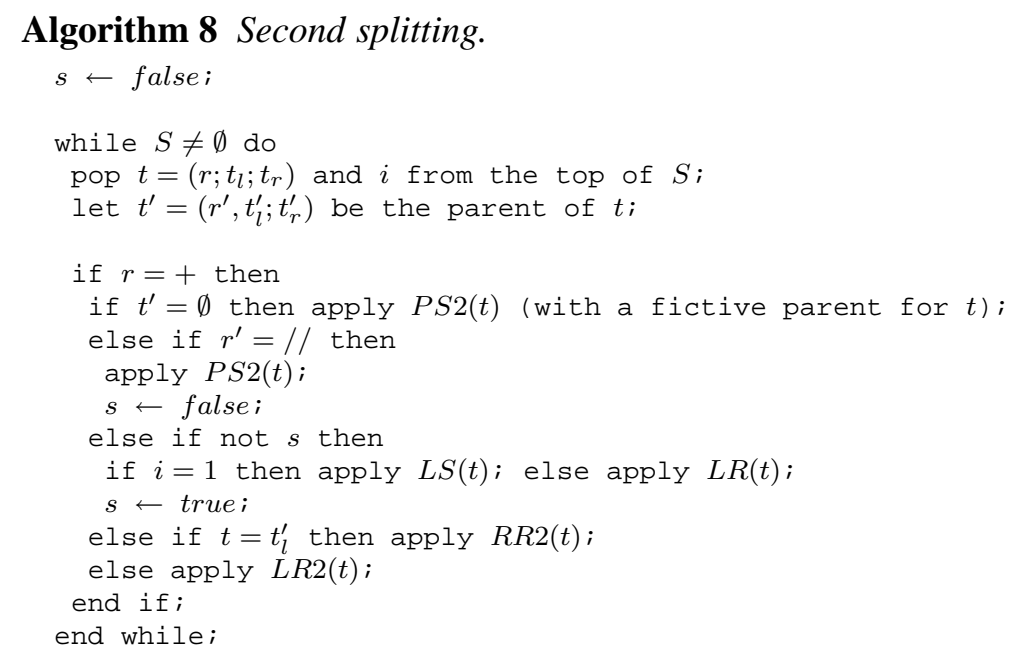

\subsection{Complexity}

Consider now the complexity of the whole splitting phase. Let $k=|D|$ and $p_{u}$ be the number of arcs of a SP-component $D_{u}$. The splitting of a SP-component $D_{v}$ into a SP-decomposition $E_{v}$ requires $O\left(p_{v}\right)$ operations for the splitting itself, $O\left(p_{v}^{3}\right)$ operations for the aggregations that follow (because, for each component $D_{w}$ of $E_{v}, O\left(p_{w}^{3}\right)$ operations are needed, but $p_{v}=\sum_{D_{w} \in E_{v}} p_{w}$, so $\left.p_{v}^{3}>\sum_{D_{w} \in E_{v}} p_{w}^{3}\right)$, and $O\left(p_{v}\right)$ operations to find the flow and the tension of each SP-component of $E_{v}$. To conclude, the complexity of a splitting is $O\left(p_{v}^{3}\right)$ operations, and it is clear that no more than $\min \{n-2 ; 2(k-1)\}$ splittings will be needed (no more than one per node and no more than 2 per SP-component of $D)$. Thus, the whole splitting phase requires $O\left(\min \{n ; 2 k\} m^{3}\right)$ operations. 


\section{Numerical Results}

Tables 1 and 2 present a practical comparison of methods, which is always difficult because of all kinds of biases. But the goal here is to get an idea of how the methods behave on QSP-graphs. Table 1 shows results when the size of the graphs varies and their SP-perturbation is set to $4 \%$. Table 2 points the performances of the methods for various SP-perturbation, the graph size being set to $n=500$ and $m=3000$. Results are expressed in seconds on a Celeron $500 \mathrm{MHz}$ processor under a Linux operating system. We used GNU C++ 2.95 compiler and its object-oriented features to implement the methods. The results are means of series of 10 tests on randomly generated graphs. Both $A$ and $B$ are fixed to 1000 . The cardinality of the SP-decomposition, for both the reduction and the path approaches, is also presented.

\begin{tabular}{|c|c|c|c|c|c|c|c|c|c|}
\hline \multirow[t]{2}{*}{ Nodes } & \multirow[t]{2}{*}{ Arcs } & \multirow[t]{2}{*}{ Kilter } & \multirow{2}{*}{$\begin{array}{c}\text { Dual } \\
\text { Cost-Scaling }\end{array}$} & \multicolumn{2}{|c|}{ Reconstruction (Total Split) } & \multicolumn{2}{|c|}{ Reconstruction (Min Split) } & \multicolumn{2}{|c|}{ Components } \\
\hline & & & & Reduction & Path & Reduction & Path & Reduction & Path \\
\hline 50 & 100 & 0.01 & 0.02 & 0.01 & 0.01 & 0.02 & 0.02 & 5 & 6 \\
\hline 50 & 200 & 0.02 & 0.03 & 0.03 & 0.03 & 0.05 & 0.05 & 9 & 10 \\
\hline 50 & 300 & 0.03 & 0.04 & 0.04 & 0.04 & 0.08 & 0.08 & 13 & 16 \\
\hline 50 & 400 & 0.04 & 0.07 & 0.06 & 0.06 & 0.12 & 0.10 & 16 & 19 \\
\hline 50 & 500 & 0.06 & 0.08 & 0.07 & 0.08 & 0.16 & 0.13 & 21 & 23 \\
\hline 100 & 200 & 0.03 & 0.06 & 0.03 & 0.03 & 0.05 & 0.05 & 12 & 11 \\
\hline 100 & 400 & 0.07 & 0.09 & 0.07 & 0.07 & 0.13 & 0.12 & 20 & 21 \\
\hline 100 & 600 & 0.10 & 0.15 & 0.12 & 0.12 & 0.20 & 0.18 & 29 & 31 \\
\hline 100 & 800 & 0.16 & 0.18 & 0.20 & 0.18 & 0.30 & 0.24 & 37 & 39 \\
\hline 100 & 1000 & 0.23 & 0.26 & 0.25 & 0.24 & 0.41 & 0.34 & 43 & 48 \\
\hline 500 & 1000 & 0.67 & 0.84 & 0.71 & 0.70 & 0.47 & 0.44 & 55 & 61 \\
\hline 500 & 2000 & 1.48 & 1.57 & 2.11 & 1.97 & 1.21 & 0.95 & 107 & 116 \\
\hline 500 & 3000 & 2.31 & 2.20 & 3.43 & 3.35 & 1.90 & 1.38 & 153 & 170 \\
\hline 500 & 4000 & 3.21 & 3.24 & 4.58 & 4.63 & 2.65 & 1.97 & 198 & 220 \\
\hline 500 & 5000 & 4.21 & 3.84 & 5.93 & 5.88 & 3.36 & 2.25 & 245 & 278 \\
\hline 1000 & 2000 & 2.34 & 2.65 & 3.35 & 3.21 & 1.41 & 1.22 & 110 & 124 \\
\hline 1000 & 4000 & 5.27 & 4.54 & 7.83 & 7.70 & 3.46 & 2.70 & 212 & 227 \\
\hline 1000 & 6000 & 8.17 & 7.16 & 12.47 & 12.78 & 5.39 & 3.95 & 310 & 344 \\
\hline 1000 & 8000 & 11.43 & 8.70 & 19.27 & 19.03 & 8.03 & 5.62 & 411 & 450 \\
\hline
\end{tabular}

Table 1: Numerical results, graph size influence.

\begin{tabular}{|c|c|c|c|c|c|c|c|c|}
\hline \multirow{2}{*}{$\begin{array}{c}\text { SP-Perturbation } \\
(\%)\end{array}$} & \multirow[t]{2}{*}{ Kilter } & \multirow{2}{*}{$\begin{array}{c}\text { Dual } \\
\text { Cost-Scaling }\end{array}$} & \multicolumn{2}{|c|}{ Reconstruction (Total Split) } & \multicolumn{2}{|c|}{ Reconstruction (Min Split) } & \multicolumn{2}{|c|}{ Components } \\
\hline & & & Reduction & Path & Reduction & Path & Reduction & Path \\
\hline 2 & 1,85 & 2,28 & 1,80 & 1,87 & 1,37 & 1,13 & 79 & 85 \\
\hline 3 & 2,02 & 2,30 & 2,62 & 2,55 & 1,68 & 1,27 & 117 & 129 \\
\hline 4 & 2,21 & 2,45 & 3,20 & 3,07 & 1,85 & 1,45 & 154 & 166 \\
\hline 5 & 2,43 & 2,34 & 3,82 & 3,70 & 2,04 & 1,55 & 196 & 211 \\
\hline 6 & 2,63 & 2,27 & 4,50 & 4,49 & 2,35 & 1,78 & 225 & 242 \\
\hline 7 & 2,80 & 2,11 & 5,38 & 5,10 & 2,66 & 2,03 & 264 & 279 \\
\hline 8 & 2,83 & 2,12 & 5,45 & 5,48 & 2,80 & 2,26 & 299 & 321 \\
\hline 9 & 3,07 & 2,11 & 6,02 & 5,68 & 3,04 & 2,42 & 333 & 360 \\
\hline 10 & 3,27 & 2,12 & 6,82 & 6,42 & 3,42 & 2,78 & 369 & 393 \\
\hline 11 & 3,31 & 2,18 & 6,86 & 6,85 & 3,49 & 2,97 & 401 & 423 \\
\hline 12 & 3,49 & 2,14 & 7,65 & 7,39 & 4,05 & 3,40 & 435 & 466 \\
\hline 13 & 3,52 & 2,16 & 7,84 & 7,60 & 4,07 & 3,59 & 467 & 499 \\
\hline 14 & 3,88 & 2,11 & 8,67 & 8,34 & 4,61 & 3,98 & 495 & 525 \\
\hline 15 & 3,86 & 2,06 & 8,94 & 8,63 & 4,81 & 4,41 & 535 & 568 \\
\hline 20 & 4,47 & 2,02 & 11,70 & 11,38 & 6,69 & 6,43 & 690 & 719 \\
\hline 30 & 5,95 & 1,91 & 17,14 & 16,06 & 11,27 & 10,72 & 1000 & 1036 \\
\hline 40 & 6,41 & 1,89 & 20,39 & 19,16 & 14,92 & 14,41 & 1305 & 1333 \\
\hline
\end{tabular}

Table 2: Numerical results, SP-perturbation influence.

The reconstruction method appears to be more efficient when the path approach is used to decompose. In fact, the reduction technique provides a more compact SP-decomposition that reveals less adapted for the reconstruction. Moreover, the minimal splitting appears to be a key to the good performances of the reconstruction algorithm. To conclude, the efficiency of the various 
methods depends on both the SP-perturbation and the size of the graph. The reconstruction method is well suited for a SP-perturbation below $8 \%$ and for large QSP-graphs, whereas the dual costscaling method is better suited for non-specific graphs or small QSP-graphs.

\section{Conclusion}

This article proposes a new algorithm to solve CPLCT on QSP-graphs that proves to be competitive with existing methods. It also describes a new way of recognizing a SP-graph and proposes two heuristic approaches to decompose a graph into SP-subgraphs. To find a "best" SPdecomposition of a graph needs to be formulated, e.g. to minimize the number of SP-components, and the complexity of the problem discussed.

\section{References}

[1] R.K. Ahuja, D.S. Hochbaum, and J.B. Orlin. Solving the Convex Cost Integer Dual Network Flow Problem. In Management Science, volume 49, pages 950-964, 2003.

[2] R.K. Ahuja, T.L. Magnanti, and J.B. Orlin. Network Flows - Theory, Algorithms, and Applications. Prentice Hall, 1993.

[3] B. Bachelet. Modélisation et optimisation de problèmes de synchronisation dans les documents hypermédia. PhD thesis, Université Blaise Pascal, Clermont-Ferrand, France, 2003.

[4] B. Bachelet and P. Mahey. Minimum Convex-Cost Tension Problems on Series-Parallel Graphs. Technical Report RR03-06, LIMOS, Université Blaise Pascal, Clermont-Ferrand, France, 2003.

[5] H.L. Bodlaender and B. de Fluiter. Parallel Algorithms for Series Parallel Graphs. In 4th Annual European Symposium on Algorithms, pages 277-289. Springer-Verlag, 1996.

[6] M.C. Buchanan and P.T. Zellweger. Specifying Temporal Behavior in Hypermedia Documents. In European Conference on Hypertext '92, pages 262-271, 1992.

[7] A.K. Datta and R.K. Sen. An Efficient Scheme to Solve Two Problems for Two-Terminal Series Parallel Graphs. In Information Processing Letters, volume 71, pages 9-15. Elsevier Science, 1999.

[8] R.J. Duffin. Topology of Series-Parallel Networks. In Journal of Mathematical Analysis and Applications, volume 10, pages 303-318, 1965.

[9] D. Eppstein. Parallel Recognition of Series-Parallel Graphs. In Information and Computation, volume 98-1, pages 41-55, 1992.

[10] J.M. Pla. An Out-of-Kilter Algorithm for Solving Minimum Cost Potential Problems. In Mathematical Programming, volume 1, pages 275-290, 1971.

[11] R.T. Rockefellar. Convex Analysis. Princeton University Press, 1970.

[12] B. Schoenmakers. A New Algorithm for the Recognition of Series Parallel Graphs. Technical report, No CS-59504, Centrum voor Wiskunde en Informatica, Amsterdam, The Netherlands, 1995.

[13] J. Valdes, R.E. Tarjan, and E.L. Lawler. The Recognition of Series Parallel Digraphs. In SIAM Journal on Computing, volume 11-2, pages 298-313, 1982. 